\title{
Interfacial Polymerization of Cellulose Nanocrystal Polyamide Janus Nanocomposites with Controlled Architectures
}

\author{
Michael S. Reid ${ }^{*}$, Johan Erlandsson ${ }^{1}$ and Lars Wågberg ${ }^{1,2 *}$ \\ ${ }^{1}$ Department of Fiber and Polymer Technology \\ Division of Fibre Technology \\ KTH Royal Institute of Technology \\ Teknikringen 56-58, 10044 Stockholm, Sweden \\ E-mail: mreid@kth.se; wagberg@kth.se \\ ${ }^{2}$ Department of Fiber and Polymer Technology \\ Wallenberg Wood Science Center \\ KTH Royal Institute of Technology \\ Teknikringen 56-58, 10044 Stockholm, Sweden
}

*Corresponding Authors: mreid@kth.se; wagberg@kth.se

Keywords: CNCs, nanocomposite, polyamide, Janus, interfacial

\begin{abstract}
The widespread use of renewable nanomaterials has been limited due to poor integration with conventional polymer matrices. Often, chemical and physical surface modifications are implemented to improve compatibility, however this comes with environmental and economic cost. This work demonstrates that renewable nanomaterials, specifically cellulose nanocrystals (CNCs), can be utilized in their unmodified state and presents a simple and versatile, one-step method to produce polyamide/CNC nanocomposites with unique Janus-like properties. Nanocomposites in the form of films, fibres and capsules are prepared by dispersing as prepared $\mathrm{CNCs}$ in the aqueous phase prior to the interfacial polymerization of aromatic diamines and acyl chlorides. The diamines in the aqueous phase not only serve as a monomer for polymerization but, additionally adsorb to, and promote the incorporation of $\mathrm{CNCs}$ into the nanocomposite. Regardless of the architecture CNCs are only present along the surface facing the aqueous phase resulting in materials with unique, Janus-like wetting behaviour and potential applications in filtration, separations, drug delivery and advanced fibres.
\end{abstract}




\section{Main Text}

Polymer nanocomposites have become a ubiquitous part of today's society. They have mostly found applications in the automotive and aerospace industries, but additionally in niche markets, such as catalysts, separators and electrolytes. ${ }^{1,2}$ However, as the global demand for sustainable and renewable materials grows, researchers are looking beyond traditional carbon-based nanoparticles (carbon black, carbon nanotubes and graphene) to renewable nanoscale materials such as nanocellulose, lignin and chitin. ${ }^{3}$

Due to their availability and projected cost, nanocellulose in the form of cellulose nanocrystals (CNC) and cellulose nanofibrils (CNFs) have received the majority of attention. ${ }^{4}$ Sourced from woods, grasses, tunicates, algae or bacteria CNCs and CNFs are extracted via acid hydrolysis or combinations of chemical and mechanical processing respectively. ${ }^{5,6}$ Exhibiting rod and fibril-like morphologies CNCs and CNFs have many advantageous mechanical, rheological and optical properties, yielding potential applications ranging from electronics and biomedical devices, to catalytic supports and coatings. ${ }^{7}$ Yet much like nanoclays, the hydrophilic nature of CNCs and CNFs limits compatibility with hydrophobic polymer matrices. Moreover, the relatively low thermal stability can lead to discolouration and degradation in traditional extrusion and injection molding processes. ${ }^{8}$ To address this, many researchers have sought to modify nanocelluloses to improve both compatibility and thermal stability. The most widely used approach is to chemically or physically graft small molecules or polymers to or from the particle surface. For example, the use of surfactants can aid dispersion in non-polar solvents, ${ }^{9,10}$ altering charged groups and selection of counter-ions can increase thermal stability, ${ }^{11-13}$ and grafting polymers improves compatibility within hydrophobic polymer matrices. ${ }^{14}$ However, as the number of modification steps and additional components increases so too does the economic cost. Moreover, due to the high specific surface area of nanocellulose the degree of substitution and molecular mass must be considerable for modifications to be effective thus, reducing the cellulose content (by mass) and the overall 
sustainability of the final material. Ideally, CNCs and CNFs should be used as unmodified materials.

As we continue to progress towards more advanced nanoscale devices, new materials and insights can yield new opportunities for old ideas. One such example is the interfacial polymerization of polyamides. While to achieve high molecular weights at the industrial scale polyamide producers have long sought to eliminate water by using batch autoclaves, vented extruders or flashing tubes ${ }^{15,16}$ the interfacial polymerization of polyamide at the oil/water interface has often been considered as a simple laboratory demonstration. Yet, interfacial polymerization is a tremendously active field and is an excellent method to prepare ultrathin films, membranes, capsules and fibres. ${ }^{17}$

Recently, there has been significant interest in using CNCs to create supported thin film polyamide composite membranes via interfacial polymerization. ${ }^{18-22}$ In these works aqueous dispersions of CNCs and diamines are deposited on an ultrafiltration membrane from which an oil phase, containing an acidic chloride, is filtered through and the resulting interfacial polymerization forms a submicron composite polyamide layer on top of the ultrafiltration membrane. Although, inclusion of CNCs generally improves flux through the membrane, and in some cases selectivity, ${ }^{20,22}$ these composites are bound to the underlying polymer support, and are limited to a single architecture.

Other high aspect ratio nanoparticles such as carbon nanotubes and graphene have been used to prepare reinforced, support free composites ${ }^{23-25}$ yet surfactants or surface functionalization are often required to form stable suspensions in either the aqueous or oil phase during preparation. Moreover, the $\mathrm{sp}^{2}$ carbon structure of carbon nanotubes and graphene does not natively support hydrogen bonding within the polyamide matrix, ${ }^{26,27}$ and surface modification is often used to improve compatability. ${ }^{28}$

To demonstrate that unmodified CNCs have potential as reinforcing agents or as functional components in composite materials herein we show the fabrication of 
polyamide/CNC nanocomposites via a one-step interfacial polymerization method. We prepare nanocomposites with different form factors including films, fibres and capsules (Figure 1) to demonstrate the simplicity and flexibility of the method. Additionally, we specifically examine the interaction between $\mathrm{CNCs}$ and diamide monomers to understand the mechanism that drives composite formation. Using this method, we create Janus-like materials that are not possible with conventional processing techniques, (extrusion, solvent casting, etc.) giving rise to materials with controlled wetting properties and potential applications as separators/barriers, drug delivery devices or as internally wicking fibres.

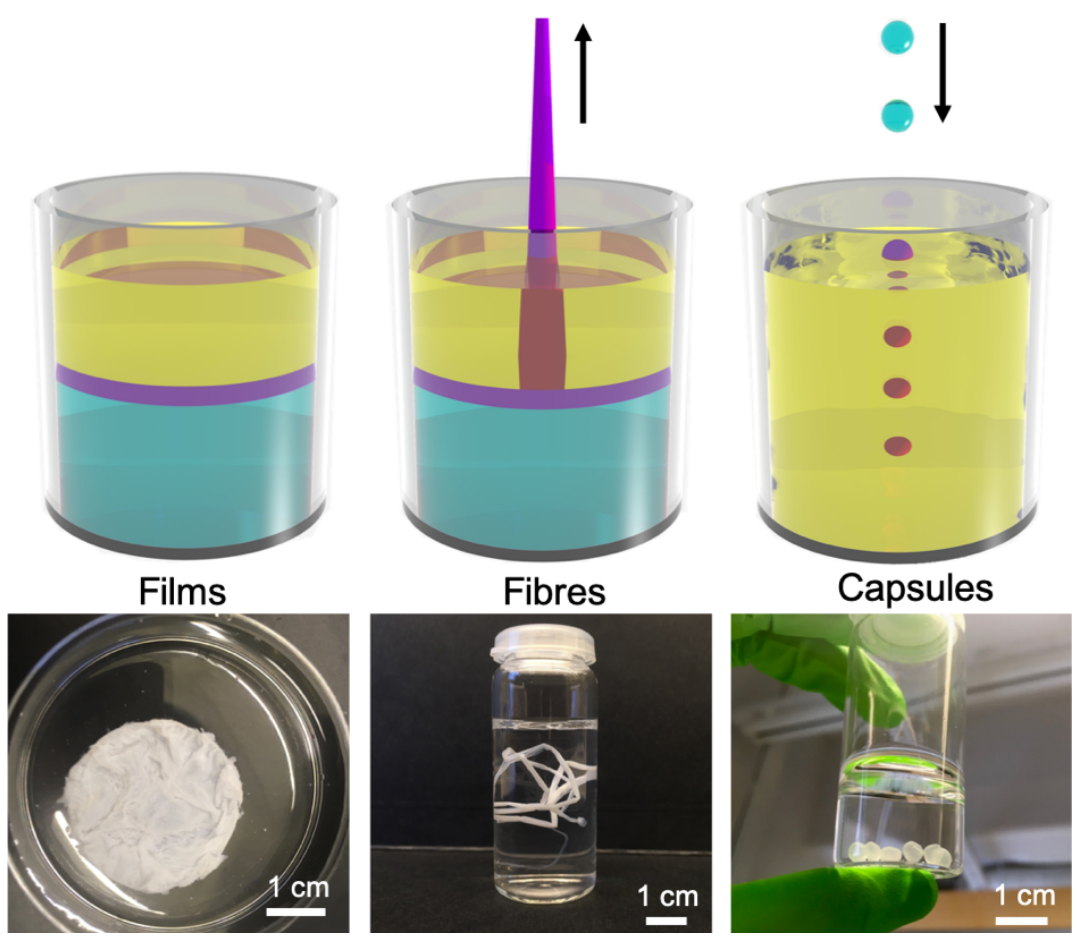

Figure 1: Schematic representation of nanocomposite formation and accompanying photographs of the nanocomposites. The oil phase containing acid chlorides is shown in yellow, the aqueous phase containing amine monomers and $\mathrm{CNCs}$ is shown in blue, and the nanocomposite is shown in purple.

Nanocomposites were prepared by combining sulfuric acid extracted $\mathrm{CNCs}^{29}$ and mxylylenediamine (MXD) in the aqueous phase (AP) and sebacoyl chloride and trimesoyl chloride (TMC) in the oil phase (OP). MXD was selected due to the interesting crystallization behaviour $^{30,31}$ and excellent barrier properties, ${ }^{32-34}$ however nanocomposites can be similarly formed using more traditional linear diamines such as hexamethylenediamine (Figure S1 
Supporting Information). Nanocomposites were formed by either layering the OP on top of the $\mathrm{AP}$ (and pulling the interface to form fibres) or by dripping the diamine/CNC dispersion through the OP to form aqueous containing capsules.

The interfacial polymerization reaction is rapid, however within this process there are several limiting steps (incipient film formation, slowdown of film formation, and diffusion limited growth) that have proved challenging to model effectively. ${ }^{35-37}$ In general, the polymerization follows a step growth mechanism, where amine monomers diffuse through the interface to condense with acidic chlorides from which, the polymers reach a high enough molecular weight to precipitate out of solution. ${ }^{38}$ As polymerization progresses the interface grows towards the OP where the thickness of the polymer layer can be controlled by altering the time of polymerization or the concentration of the monomers in solution (Figure S2 Supporting information).

Inclusion of CNCs into the final composite structure requires CNCs to either diffuse to or be drawn to the interface, such that they can be incorporated during polymerization. CNCs are well known to be good Pickering emulsion stabilizers however, they often require significant energy input via sonication to migrate to the oil/water interface. ${ }^{39,40}$ When small molecules, such as surfactants are adsorbed, they can more effectively migrate to the interface, ${ }^{41,42}$ yet inclusion of additional components increases economic and environmental costs in comparison to native materials. For nanocomposite films where the polymerization is relatively static (i.e. the reaction is left undisturbed until completion) CNCs have sufficient time to diffuse to and interact with interface. This is in contrast to fibre formation, where the drawing process continually creates new interfaces to which CNCs must rapidly migrate in order to be incorporated in the nanocomposite. As a result, we aim to use diamine molecules to perform two distinct functions during composite formation: i) serve as monomers for polymerization and ii) adsorb to CNCs to promote migration to the oil/water interface. 
Adsorption behaviour was investigated upon the addition of MXD to CNC dispersions. Zeta potential measurements (Table 1) and isothermal titration calorimetry (ITC) (Figure 2) show that MXD adsorbs to the CNC surface and thus promotes particle migration and incorporation during polymerization. Notably, adsorption is observed at both $\mathrm{pH} 10.5$ and $\mathrm{pH}$ 3, above and below the $\mathrm{pK}_{\mathrm{a}}$ of $\mathrm{MXD}\left(\mathrm{pK}_{\mathrm{a}} \sim 9.2\right)$ respectively. ${ }^{43}$ Fitting the ITC curves to a generic binding process with independent sites indicates that MXD spontaneously adsorbs to the $\mathrm{CNC}$ surface $(\Delta \mathrm{G}<0)$ however, the mode of adsorption is dependent on $\mathrm{pH}^{44}$ (See Supporting information for ITC fitting details) At $\mathrm{pH}$ 3, MXD is cationic and thus adsorption is entropically driven through the release of counter ions with the anionic sulfate half esters on the CNC surface. However, significantly above the $\mathrm{pK}_{\mathrm{a}} \mathrm{MXD}$ is no longer charged and adsorption occurs via an enthalpy driven process, suggesting that while specific interactions between MXD and CNCs do not drive adsorption, they do play a role. Adsorption at both high and low $\mathrm{pH}$ is beneficial as the $\mathrm{pH}$ is expected to vary throughout the interfacial polymerization. Specifically, during the initial phases of polymerization, high monomer concentration in the AP (2 wt.\%) yields a $\mathrm{pH}$ above the $\mathrm{pK}_{\mathrm{a}}$ of $\mathrm{MXD}$. As the polymerization progresses, condensation of the amine and acidic chlorides produces $\mathrm{HCl}$ which locally reduces the $\mathrm{pH}$. While adsorption will primarily occur during initial mixing of MXD and CNCs, from the ITC and zeta potential data, we do not expect desorption to occur as polymerization progresses.

Table 1: Zeta potential and apparent particle size measured by DLS for CNC and MXD dispersions.

\begin{tabular}{llll}
\hline $\begin{array}{l}\text { [MXD] } \\
\text { (wt.\%) }\end{array}$ & pH & $\begin{array}{l}\text { Zeta } \\
\text { potential }(\mathrm{mV})\end{array}$ & $\begin{array}{l}\text { Apparent } \\
\text { Particle Size } \\
(\mathrm{nm})\end{array}$ \\
\hline 0 & 6.5 & $-44 \pm 4$ & $75 \pm 1$ \\
0.125 & 6.5 & $-17 \pm 1$ & $74 \pm 1$ \\
1.0 & 11.0 & $-22 \pm 2$ & $76 \pm 1$
\end{tabular}



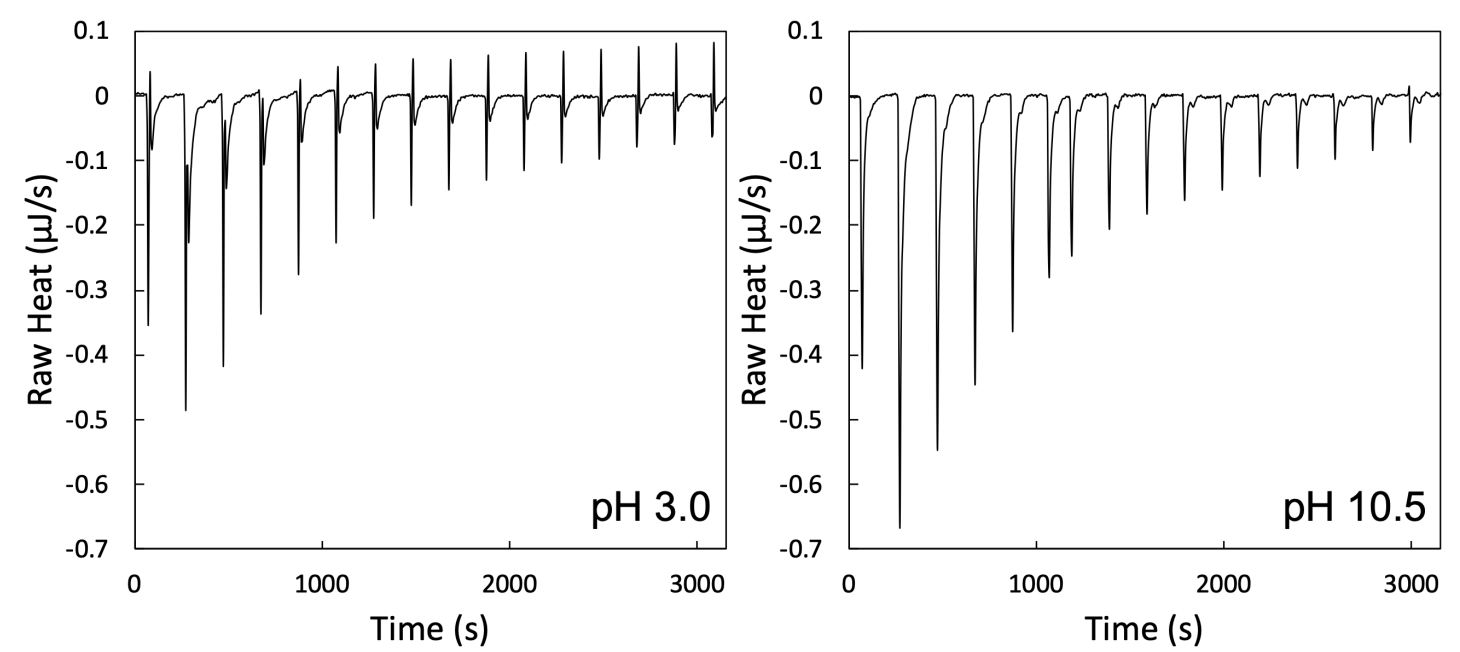

Figure 2: Baseline corrected raw heats for $0.1 \mathrm{wt} \% \mathrm{MXD}$ injected into $1 \mathrm{wt} \% \mathrm{CNC}$ dispersion at $\mathrm{pH} 3$ and $\mathrm{pH} 10.5$

Due to adsorption, CNCs can potentially alter the interfacial polymerization as it is dependent on both the diffusivity of MXD and the interfacial tension at the oil/water interface. ${ }^{17}$ Interfacial tension measurements between aqueous solutions of MXD and dodecane show that above 1 wt.\% MXD the interface becomes saturated and CNCs do not significantly impact the interfacial tension between the two phases. (Figure S4 Supporting Information) As a result, it is expected that CNCs do not have a significant effect on the polymerization of the nanocomposite. This is supported by results in Figure 3a and Figure 3d where nanocomposite films with 0 wt. $\%$ and $0.5 \mathrm{wt} . \% \mathrm{CNCs}$, the lowest and highest concentrations investigated, have similar thicknesses and structure when imaged by SEM. The impact of CNCs on polymerization is further investigated by ATR-FTIR spectroscopy and is discussed below. 

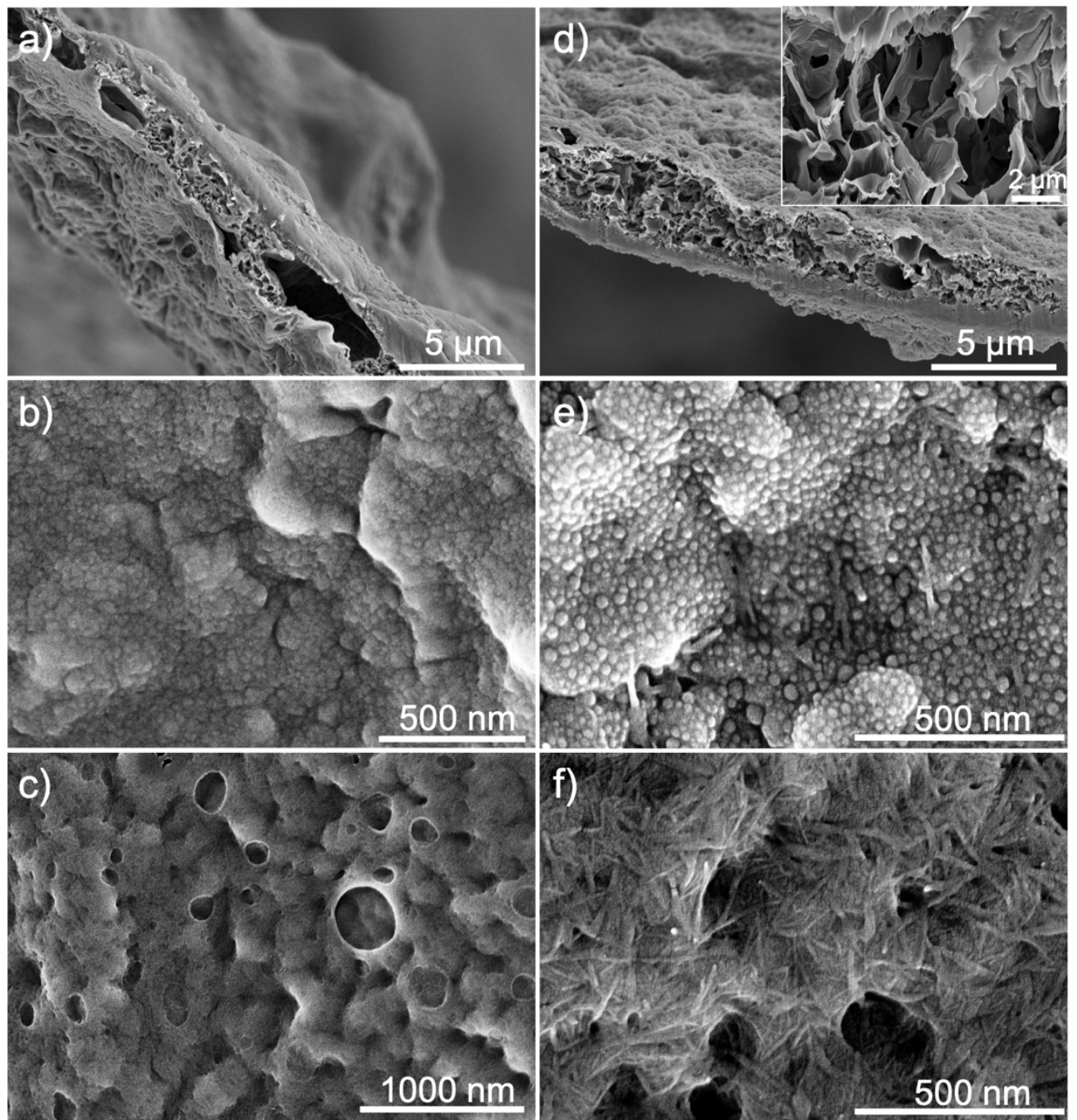

Figure 3: Scanning electron microscopy images of $\mathrm{MXD} / \mathrm{CNC}$ nanocomposite films formulated with 2 wt.\% MXD, 0.25 wt.\% TMC and (a-c) 0 wt.\% CNC and (d-f) 0.5 wt.\% CNC. The images show the edge, OP surface, and AP surface from top to bottom respectively. Inset in (d) shows interior pore morphology.

The interior structure of the nanocomposite is highly porous with a cellular-like morphology, typical of interfacially polymerized membranes and films. ${ }^{45}$ Each cell is separated by thin polymer walls that are void of CNCs (Figure 3d inset). Currently, the connectivity between the cells is unclear however, the morphology and selectivity of the membrane can potentially be tuned by altering monomer type and concentration. ${ }^{46}$ Examining the surface of the nanocomposite reveals that CNCs are incorporated largely only at the surface facing the AP where they form a dense network of overlapping particles that lay parallel to the nanocomposite surface (Figure 3f). This can be further seen in atomic force microscopy images (Figure S5 
Supporting Information). In contrast, few or no CNCs are observed at the oil facing surface. We propose this Janus-like structure results from the rapid polymerization of the polyamide at the interface, whereby the initial film formation limits CNCs from penetrating deeper into the composite structure. As a result, $\mathrm{CNCs}$ have little impact on the surface facing the oil phase, with both native and $\mathrm{CNC}$ modified materials exhibiting rough, globular like structures, typical of polyamides formed by interfacial polymerization. ${ }^{47}$ Moreover, with CNCs only present at the surface facing the AP they constitute a relatively small percentage of the overall composite mass and have limited impact on the thermal properties of the nanocomposite, as measured by thermogravimetric analysis (Figure S6 Supporting Information).

Similar to the films both the fibre and capsule nanocomposites show Janus-like morphology with different interior and exterior structures (Figure 4). Specifically, as the fibres are pulled out of the interface a tubular structure is formed, in which the interior and exterior surfaces face the AP and OP, respectively, and thus confine CNCs to the interior of the fibre (Figure 4d). CNCs were observed along the entire length of the fibre, demonstrating that even though new interface is rapidly created as the fibre is drawn from solution, the CNCs are sufficiently mobile in the AP to be incorporated during polymerization. The unique structure of these fibres, have potential applications as internally wicking fibres or as hollow fibre membranes for filtration applications. 


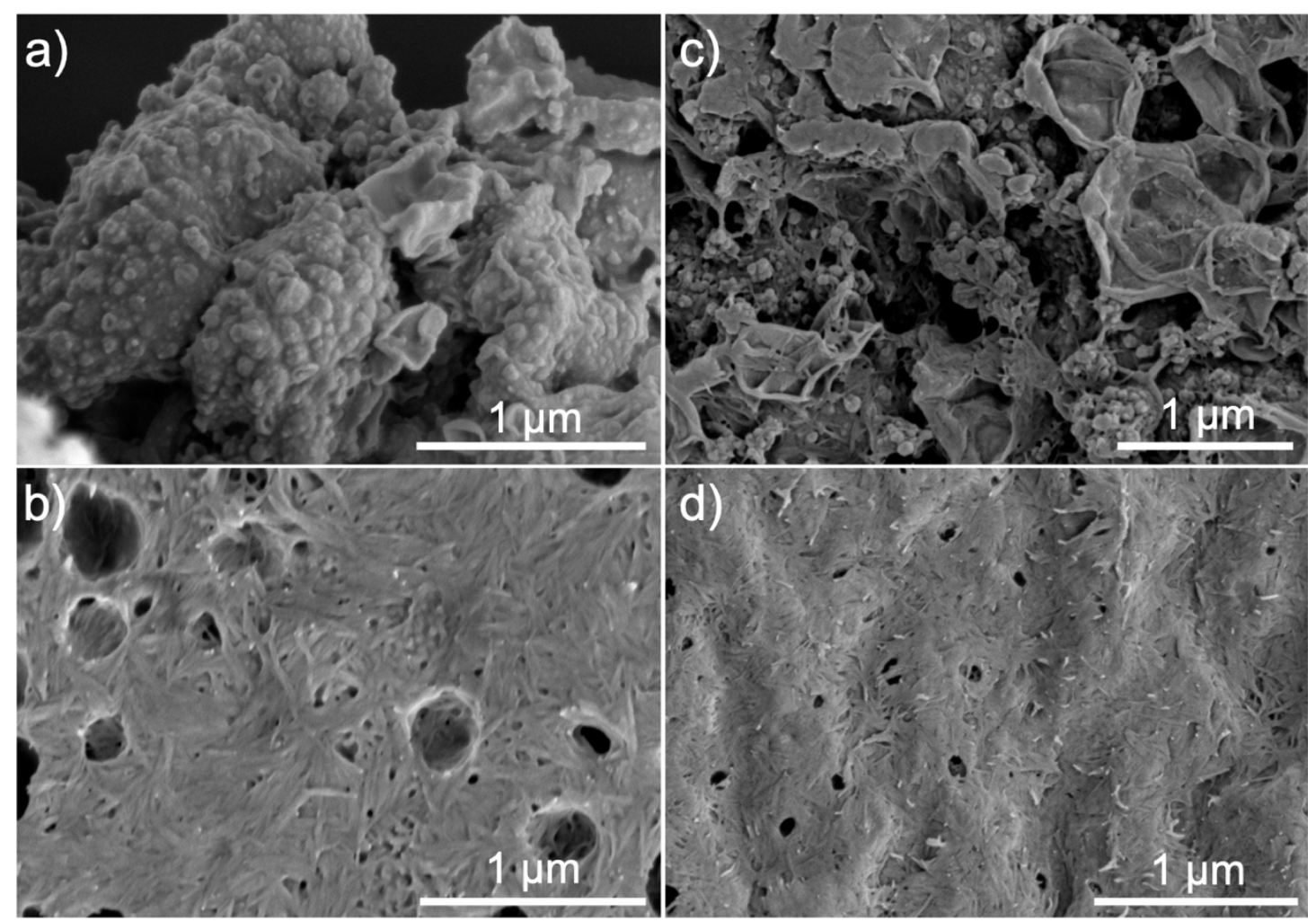

Figure 4: Scanning electron microscopy images of MXD/CNC nanocomposite (a, b) capsules and (c, d) fibres formulated with $2 \mathrm{wt} . \% \mathrm{MXD}, 0.25 \mathrm{wt} . \% \mathrm{TMC}$ and $0.5 \mathrm{wt} . \% \mathrm{CNC}$. The images show the (a,c) OP surfaces and $(\mathbf{b}, \mathbf{d})$ AP surface of the nanocomposites.

During the formation of the capsules rapid polymerization around the droplet creates a polyamide shell which contains the AP and CNCs. Similar to the nanocomposite films, amine monomers seem to diffuse through the capsule wall and continue to polymerize with the TMC in the OP. As a result, the capsule wall is highly porous with a cellular morphology (Figure S7 Supporting Information) and CNCs are only present at AP surface, as detected for the films and the fibres. Regardless of the formulation method, the resultant nanocomposites incorporate CNCs only at the surface facing the AP, creating unique Janus-like architectures.

While the CNCs are only present at the AP surface, a sufficient number are incorporated into the nanocomposite such that they impact the overall material properties and performance as evidenced by ATR FTIR spectroscopy and contact angle measurements. For all nanocomposite films, ATR FTIR spectra of the AP surface show characteristic aromatic polyamide bands at $1633 \mathrm{~cm}^{-1}, 1524 \mathrm{~cm}^{-1}, 1425 \mathrm{~cm}^{-1}$ and $1281 \mathrm{~cm}^{-1}$, attributed to $v(\mathrm{C}=\mathrm{O}), \delta(\mathrm{N}-$ 
$\mathrm{H}), \delta\left(\mathrm{C}-\mathrm{H}_{2}\right)$ and $\delta(\mathrm{C}-\mathrm{H})$ vibrational modes respectively (Figure 5a). ${ }^{34,48}$ The intensity and width of these bands remain relatively unchanged with the addition of $\mathrm{CNCs}$, indicating that the polymerization is largely unaffected by CNCs, in good agreement with SEM images. However, evidence of CNCs within the nanocomposite structure is observed by the development of peaks at $1057 \mathrm{~cm}^{-1}$ and $1033 \mathrm{~cm}^{-1}$. These peaks are assigned to $v(\mathrm{C}-\mathrm{O})$ at the $\mathrm{C} 6$ and $\mathrm{C} 3$ position of cellulose, respectively, and show an increase in signal intensity with $\mathrm{CNC}$ concentration. ${ }^{49} \mathrm{In}$ addition to the characteristic cellulose $v(\mathrm{C}-\mathrm{O})$ peaks, CNCs are observed at higher vibrational frequencies; specifically within the $v(\mathrm{C}-\mathrm{H})$ and $v(\mathrm{O}-\mathrm{H})$ regions of the spectra (Figure $5 \mathrm{~b}$ ). Broadening of the peak at $2925 \mathrm{~cm}^{-1}$ and development of band shoulders at $2967 \mathrm{~cm}^{-1}$ indicate that additional $v(\mathrm{C}-\mathrm{H})$ modes, resulting from $\mathrm{CNCs}$, are present for the nanocomposite. Furthermore, Figure $5 \mathrm{~b}$ shows that with increasing $\mathrm{CNC}$ concentration a new peak develops at $3664 \mathrm{~cm}^{-1}$. While, $v(\mathrm{O}-\mathrm{H})$ modes from the alcohol groups within cellulose range from $3200-$ $3500 \mathrm{~cm}^{-1},{ }^{49}$ the vibrational modes from $3700-3600 \mathrm{~cm}^{-1}$ are attributed to weakly bound or free $v(\mathrm{O}-\mathrm{H})$ modes and are typically observed within highly structured clays. ${ }^{50}$ The development of this peak suggests that when CNCs are incorporated into the nanocomposite, a portion of the alcohol groups cannot effectively hydrogen bond with other $\mathrm{CNCs}$ or the N-H within the polyamide, and can be speculated to be due to an immobilization of the CNCs parallel to the nanocomposite surface. Additionally, ATR FTIR spectra of the OP surface (Figure S8 Supporting Information) show no characteristic cellulose bands and further demonstrates that CNCs are only present at the AP surface. 

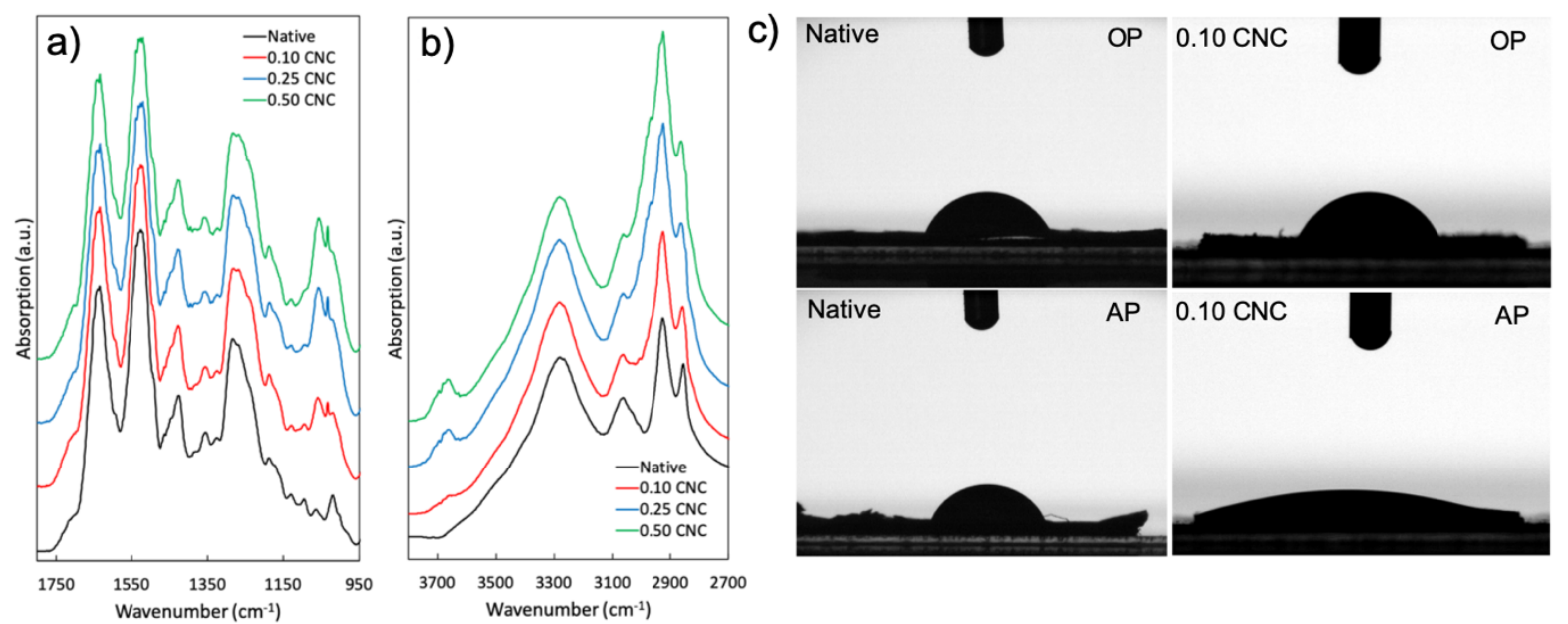

Figure 5: ATR FTIR spectra of polyamide CNC nanocomposite films from a) $1800-950 \mathrm{~cm}^{-1}$ showing the amide and $\mathrm{CNC}$ regions and b) $3800-2800 \mathrm{~cm}^{-1}$ displaying the $\mathrm{O}-\mathrm{H}, \mathrm{N}-\mathrm{H}$ and $\mathrm{C}-\mathrm{H}$ stretching regions (c) Contact angle measurements of polyamide $\mathrm{CNC}$ nanocomposite films at the oil facing surface (OP) and aqueous facing surface (AP) of native and $0.1 \mathrm{wt} . \% \mathrm{CNC}$.

As shown in Figure 5c CNCs significantly alter the wetting behaviour of the polyamide nanocomposites. Native films have a contact angle of $57 \pm 10^{\circ}$ for both OP and AP surfaces, demonstrating that the different morphology, observed by SEM (Figure 3), has little impact on the wetting behaviour. Comparatively, nanocomposites containing CNCs wet significantly more at the AP surface, with 0.1 wt. \% CNC yielding the lowest contact angle of $14 \pm 7^{\circ}$ (Figure 5c). See Table S2 in the Supporting Information for complete list of contact angles. The presence of hydrophilic CNCs only at the AP surface creates a higher surface energy in comparison to the aromatic polyamide at the OP surface, resulting in Janus-like films. While not specifically investigated in this work this controlled wetting behaviour has potential to increase flux and specificity for filtering and separation applications. Moreover, incorporating CNCs into the nanocomposites provides an opportunity to graft specific moieties, such as enzymes, antibodies, or ligands directly into the nanocomposite. ${ }^{51}$

In conclusion, we have demonstrated a versatile one-step method for producing nanocomposites via interfacial polymerization of polyamides. This procedure makes use of unmodified CNCs and is capable of producing various architectures including films, fibres and capsules, with unique Janus-like structures and properties. The formation of nanocomposites 
occurs initially via amine adsorption to the CNC surface in the aqueous phase, from which CNCs are incorporated into the nanocomposite during polymerization. Amine adsorption was observed to occur above and below the $\mathrm{pK}_{\mathrm{a}}$ of the monomer via zeta potential and ITC measurements and promotes migration of $\mathrm{CNCs}$ to the oil/water interface. The resulting nanocomposites, were found to have a Janus-like structure with CNCs only present at the AP surface as observed via SEM, AFM and ATR FTIR. Immobilizing CNCs at the nanocomposite surface created films with unique Janus-like wetting behaviour, with only the AP surface becoming more hydrophilic. While this simple one step process has been demonstrated for aromatic amides, material properties can be further tuned by altering both the aqueous and oil

phase monomers. Moreover, this methodology goes beyond filtering and separations, to potential applications including drug delivery via capsules and advanced fibres with core shell morphologies. Additionally, modification of the CNCs prior to or post polymerization has the potential to impart specific functionalities and material properties.

\section{Supporting Information}

The Supporting Information is available free of charge on the ACS Publications website. Materials and Methods, AFM and SEM images showing composite morphology, ITC data and fitting, interfacial tension data, TGA, FTIR characterization and contact angles.

\section{Acknowledgements}

Funding from the Natural Sciences and Engineering Research Council of Canada, Postdoctoral Fellowship is gratefully acknowledged. We additionally would like to thank Tomas Nyman and the Biophysical Protein Characterization Platform at the Karolinska Institutet for access to and expertise in ITC. Lars Wågberg also acknowledges The Knut and Alice Wallenberg foundation for financial support through the Wallenberg Wood Science Centre. 


\section{References}

(1) Kumar, S. K.; Benicewicz, B. C.; Vaia, R. A.; Winey, K. I. 50th Anniversary Perspective: Are Polymer Nanocomposites Practical for Applications? Macromolecules 2017, 50, 714-731.

(2) Winey, K. I.; Vaia, R. A. Polymer Nanocomposites. MRS Bull. 2007, 32, 314-322.

(3) Mishra, R. K.; Ha, S. K.; Verma, K.; Tiwari, S. K. Recent Progress in Selected BioNanomaterials and Their Engineering Applications: An Overview. J. Sci. Adv. Mater. Devices 2018, 3, 263-288.

(4) Cowie, J.; Bilek, E. M.; Wegner, T. H. Market Projections of Cellulose NanomaterialEnabled Products - Part 2: Volume Estimates. TAPPI J. 2014, 13, 57-69.

(5) Nechyporchuk, O.; Belgacem, M. N.; Bras, J. Production of Cellulose Nanofibrils: A Review of Recent Advances. Industrial Crops and Products. Elsevier B.V. December 2015, pp 2-25.

(6) Foster, E. J.; Moon, R. J.; Agarwal, U. P.; Bortner, M. J.; Bras, J.; Camarero-Espinosa, S.; Chan, K. J.; Clift, M. J. D.; Cranston, E. D.; Eichhorn, S. J.; et al. Current Characterization Methods for Cellulose Nanomaterials. Chem. Soc. Rev. 2018, 47, 2609-2679.

(7) Grishkewich, N.; Mohammed, N.; Tang, J.; Tam, K. C. Recent Advances in the Application of Cellulose Nanocrystals. Curr. Opin. Colloid Interface Sci. 2017, 29, 32 45.

(8) Venkatraman, P.; Gohn, A. M.; Rhoades, A. M.; Foster, E. J. Developing High Performance PA 11/Cellulose Nanocomposites for Industrial-Scale Melt Processing. Compos. Part B Eng. 2019, 174, 106988.

(9) Siqueira, G.; Bras, J.; Dufresne, A. Cellulosic Bionanocomposites: A Review of Preparation, Properties and Applications. Polymers (Basel). 2010, 2, 728-765.

(10) Heux, L.; Chauve, G.; Bonini, C. Nonflocculating and Chiral-Nematic Self-Ordering of Cellulose Microcrystals Suspensions in Nonpolar Solvents. Langmuir 2000, 16, 82108212.

(11) Vanderfleet, O. M.; Reid, M. S.; Bras, J.; Heux, L.; Godoy-Vargas, J.; Panga, M. K. R.; Cranston, E. D. Insight into Thermal Stability of Cellulose Nanocrystals from New Hydrolysis Methods with Acid Blends. Cellulose 2018, 7.

(12) Camarero Espinosa, S.; Kuhnt, T.; Foster, E. J.; Weder, C. Isolation of Thermally Stable Cellulose Nanocrystals by Phosphoric Acid Hydrolysis. Biomacromolecules 2013, 14, 1223-1230.

(13) Fox, D. M.; Rodriguez, R. S.; Devilbiss, M. N.; Woodcock, J.; Davis, C. S.; Sinko, R.; Keten, S.; Gilman, J. W. Simultaneously Tailoring Surface Energies and Thermal Stabilities of Cellulose Nanocrystals Using Ion Exchange: Effects on Polymer Composite Properties for Transportation, Infrastructure, and Renewable Energy Applications. ACS Appl. Mater. Interfaces 2016, 8, 27270-27281.

(14) Wohlhauser, S.; Delepierre, G.; Labet, M.; Morandi, G.; Thielemans, W.; Weder, C.; Zoppe, J. O. Grafting Polymers from Cellulose Nanocrystals: Synthesis, Properties, and Applications. Macromolecules 2018, 51, 6157-6189.

(15) Russell, S.; Robertson, D.; Lee, J. Control of Product Quality for Batch Nylon 6, 6 Autoclaves. Chem. Eng. 1998, 53.

(16) Pimentel, R. O.; Giudici, R. Simulation Model of Polyamide-6,6 Polymerization in a Continuous Two-Phase Flow Coiled Tubular Reactor. Ind. Eng. Chem. Res. 2006, 45, 4558-4566.

(17) Raaijmakers, M. J. T.; Benes, N. E. Current Trends in Interfacial Polymerization 
Chemistry. Prog. Polym. Sci. 2016, 63, 86-142.

(18) Wang, J. J.; Yang, H. C.; Wu, M. B.; Zhang, X.; Xu, Z. K. Nanofiltration Membranes with Cellulose Nanocrystals as an Interlayer for Unprecedented Performance. J. Mater. Chem. A 2017, 5, 16289-16295.

(19) Bai, L.; Liu, Y.; Bossa, N.; Ding, A.; Ren, N.; Li, G.; Liang, H.; Wiesner, M. R. Incorporation of Cellulose Nanocrystals (CNCs) into the Polyamide Layer of ThinFilm Composite (TFC) Nanofiltration Membranes for Enhanced Separation Performance and Antifouling Properties. Environ. Sci. Technol. 2018, 52, 1117811187.

(20) Smith, E.; Hendren, K.; Haag, J.; Foster, E.; Martin, S. Functionalized Cellulose Nanocrystal Nanocomposite Membranes with Controlled Interfacial Transport for Improved Reverse Osmosis Performance. Nanomaterials 2019, 9, 125.

(21) Asempour, F.; Emadzadeh, D.; Matsuura, T.; Kruczek, B. Synthesis and Characterization of Novel Cellulose Nanocrystals-Based Thin Film Nanocomposite Membranes for Reverse Osmosis Applications. Desalination 2018, 439, 179-187.

(22) Park, C. H.; Jeon, S.; Park, S. H.; Shin, M. G.; Park, M. S.; Lee, S. Y.; Lee, J. H. Cellulose Nanocrystal-Assembled Reverse Osmosis Membranes with High Rejection Performance and Excellent Antifouling. J. Mater. Chem. A 2019, 7, 3992-4001.

(23) Takeuchi, K.; Takizawa, Y.; Kitazawa, H.; Fujii, M.; Hosaka, K.; Ortiz-Medina, J.; Morelos-Gomez, A.; Cruz-Silva, R.; Fujishige, M.; Akuzawa, N.; et al. Salt Rejection Behavior of Carbon Nanotube-Polyamide Nanocomposite Reverse Osmosis Membranes in Several Salt Solutions. Desalination 2018, 443, 165-171.

(24) Kang, M.; Myung, S. J.; Jin, H. J. Nylon 610 and Carbon Nanotube Composite by in Situ Interfacial Polymerization. Polymer (Guildf). 2006, 47, 3961-3966.

(25) Jeong, J.-Y.; Lee, H.-J.; Kang, S.-W.; Tan, L.-S.; Baek, J.-B. Nylon 610/Functionalized Multiwalled Carbon Nanotube Composite Prepared from in-Situ Interfacial Polymerization. J. Polym. Sci. Part A Polym. Chem. 2008, 46, 6041-6050.

(26) Alarcón, L. M.; Malaspina, D. C.; Schulz, E. P.; Frechero, M. A.; Appignanesi, G. A. Structure and Orientation of Water Molecules at Model Hydrophobic Surfaces with Curvature: From Graphene Sheets to Carbon Nanotubes and Fullerenes. Chem. Phys. 2011, 388, 47-56.

(27) Akaishi, A.; Yonemaru, T.; Nakamura, J. Formation of Water Layers on Graphene Surfaces. ACS Omega 2017, 2, 2184-2190.

(28) Spitalsky, Z.; Tasis, D.; Papagelis, K.; Galiotis, C. Carbon Nanotube-Polymer Composites: Chemistry, Processing, Mechanical and Electrical Properties. Prog. Polym. Sci. 2010, 35, 357-401.

(29) Reid, M. S.; Villalobos, M.; Cranston, E. D. Benchmarking Cellulose Nanocrystals: From the Laboratory to Industrial Production. Langmuir 2017, 33, 1583-1598.

(30) Doudou, B. B.; Dargent, E.; Grenet, J. Crystallization and Melting Behaviour of Poly(m-Xylene Adipamide). J. Therm. Anal. Calorim. 2006, 85, 409-415.

(31) Saiter, J. M.; Arnoult, M.; Saiter, A.; Pareige, C.; Dargent, E. Crystallisation and Molecular Mobilities in Liquid and Glassy States of a MXD6 Polyamide. Compos. Interfaces 2006, 13, 403-413.

(32) Hu, Y. S.; Mehta, S.; Schiraldi, D. A.; Hiltner, A.; Baer, E. Effect of Water Sorption on Oxygen-Barrier Properties of Aromatic Polyamides. J. Polym. Sci. Part B Polym. Phys. 2005, 43, 1365-1381.

(33) Asiri, A. M. Improved Photochromic and Fatigue Performance Furylethylidene ) Succinicanhydride Doped in Polyurethane Thin Film. Polym. Eng. Sci. 2011, i, 1-5.

(34) Jia, L.; Su, G.; Yuan, Q.; Zhang, X.; Zhou, T. Difference in the Micro-Dynamics Mechanism between Aromatic Nylon and Aliphatic Nylon during Water Absorption: Spectroscopic Evidence. Phys. Chem. Chem. Phys. 2018, 20, 26764-26776. 
(35) Dhumal, S. S.; Wagh, S. J.; Suresh, A. K. Interfacial Polycondensation-Modeling of Kinetics and Film Properties. J. Memb. Sci. 2008, 325, 758-771.

(36) Karode, S. K.; Kulkarni, S. S.; Suresh, A. K.; Mashelkar, R. A. Molecular Weight Distribution in Interfacial Polymerization - Model Development and Verification. Chem. Eng. Sci. 1997, 52, 3243-3255.

(37) Freger, V. Kinetics of Film Formation by Interfacial Polycondensation. Langmuir 2005, 21, 1884-1894.

(38) Zhang, Y.; Benes, N. E.; Lammertink, R. G. H. Visualization and Characterization of Interfacial Polymerization Layer Formation. Lab Chip 2015, 15, 575-580.

(39) Kalashnikova, I.; Bizot, H.; Bertoncini, P.; Cathala, B.; Capron, I. Cellulosic Nanorods of Various Aspect Ratios for Oil in Water Pickering Emulsions. Soft Matter 2013, 9, 952.

(40) Kalashnikova, I.; Bizot, H.; Cathala, B.; Capron, I. Modulation of Cellulose Nanocrystals Amphiphilic Properties to Stabilize Oil/Water Interface.

Biomacromolecules 2012, 13, 267-275.

(41) Hu, Z.; Ballinger, S.; Pelton, R.; Cranston, E. D. Surfactant-Enhanced Cellulose Nanocrystal Pickering Emulsions. J. Colloid Interface Sci. 2015, 439, 139-148.

(42) Kedzior, S. A.; Marway, H. S.; Cranston, E. D. Tailoring Cellulose Nanocrystal and Surfactant Behavior in Miniemulsion Polymerization. Macromolecules 2017, 50, 2645-2655.

(43) Mitsubishi Gas Chemical Company Inc. "1,3-Bis(Aminomethyl)Benzene” OECD SIDS Initial Assessment Report; 2001.

(44) Lombardo, S.; Thielemans, W. Thermodynamics of Adsorption on Nanocellulose Surfaces. Cellulose 2019, 26, 249-279.

(45) Jin, Y.; Su, Z. Effects of Polymerization Conditions on Hydrophilic Groups in Aromatic Polyamide Thin Films. J. Memb. Sci. 2009, 330, 175-179.

(46) Perignon, C.; Ongmayeb, G.; Neufeld, R.; Frere, Y.; Poncelet, D. Microencapsulation by Interfacial Polymerisation: Membrane Formation and Structure. J. Microencapsul. 2015, 32, 1-15.

(47) Karan, S.; Jiang, Z.; Livingston, A. G. Sub-10 Nm Polyamide Nanofilms with Ultrafast Solvent Transport for Molecular Separation. Science (80-. ). 2015, 348, 1347-1351.

(48) Umemura, J.; Murata, Y.; Tsunashima, K.; Koizumi, N. Polarized Infrared Spectra of Poled Aromatic Polyamide Films. J. Polym. Sci. Part B Polym. Phys. 1999, 37, 531538.

(49) Maréchal, Y.; Chanzy, H. The Hydrogen Bond Network in I $\beta$ Cellulose as Observed by Infrared Spectrometry. J. Mol. Struct. 2000, 523, 183-196.

(50) Madejová, J. FTIR Techniques in Clay Mineral Studies. Vib. Spectrosc. 2003, 31, 110 .

(51) Liu, Y.; Chen, J. Y. Enzyme Immobilization on Cellulose Matrixes. J. Bioact. Compat. Polym. 2016, 31, 553-567. 